significant association emerged in particular between the presence of NAC and the active videocapillaroscopy pattern (OR 6.23; 95\% Cl: 1.058-36.71, $\mathrm{p}=0.043)$.

Conclusion: Though current data in the literature on this topic are poor, cardiac autonomic neuropathy is among the clinical manifestations of SSc. In our study population, though the limited sample size, we observed a high percentage of patients with autonomic cardiac neuropathy, which seems much more frequent with the increase in the duration of disease and based on the type of videocapillaroscopy pattern.

References:

[1] Ferri C, Emdin M, Giuggioli D, Carpeggiani C, Maielli M, Varga A, Michelassi C, Pasero G, L'Abbate A. Autonomic dysfunction in systemic sclerosis: time and frequency domain 24 hour heart rate variability analysis. $\mathrm{Br} \mathrm{J}$ Rheumatol. 1997

[2] Adler BL, Russell JW, Hummers LK, McMahan ZH. Symptoms of Autonomic Dysfunction in Systemic Sclerosis Assessed by the COMPASS-31 Questionnaire. J Rheumatol. 2018 Aug;45(8):1145-1152. doi: 10.3899/jrheum.170868. Epub 2018 Jun

Disclosure of Interests: None declared

DOI: 10.1136/annrheumdis-2020-eular.4654

\section{AB0161 CLONAL HEMATOPOIESIS IS INCREASED AND NOT RELATED TO AGING IN SYSTEMIC SCLEROSIS}

L. Ricard ${ }^{1}$, P. Hirsch ${ }^{1}$, M. Mohty ${ }^{1}$, O. Fain ${ }^{1}$, B. Gaugler ${ }^{1}$, J. Rossignol ${ }^{1}$,

F. Delhommeau ${ }^{1}$, A. Mekinian ${ }^{1}$ on behalf of na. ${ }^{1}$ qsd, qsd, France

Background: Systemic sclerosis (SSc) is an autoimmune disease characterized by fibrosis, microangiopathy and immune dysfunctions including dysregulation of proinflammatory cytokines. Clonal hematopoiesis of indeterminate potential (CHIP) is defined by the acquisition of somatic mutations in hematopoietic stem cells leading to detectable clones in the blood. Recent data have shown a higher risk of cardiovascular disease in patients with CHIP resulting from increased production of proinflammatory cytokines and accelerated atherosclerosis. Eventual links between CHIP and autoimmune diseases are undetermined.

Objectives: The aim of our study was to evaluate the prevalence of CHIP in SSc patients and its association with clinical phenotype.

Methods: Forty-one genes frequently mutated in myeloid malignancies were sequenced in peripheral blood mononuclear cells from $90 \mathrm{SSc}$ patients and from 44 healthy donors.

Results: A total of 15 somatic variants was detected in 13/90 SSc patients $(14 \%)$ and 4 somatic variants in $4 / 44(9 \%) \mathrm{HD}(\mathrm{p}=0.58)$. The prevalence of CHIP was significantly higher in younger SSc patients than in HD: $25 \%(6 / 24)$ vs $4 \%$ $(1 / 26)(p=0.045)$ under 50 years and $17 \%(7 / 42)$ vs $3 \%(1 / 38)(p=0.065)$ under 60 years. The prevalence of CHIP in patients over 70 years was similar in SSc patients and healthy donors

For SSc patients the most common mutations occurred in DNMT3A (7 variants). Other variants involved ATM, SF3B1, SETBP1, TET2, TP53, NF1 or CBL. The distribution of gene mutations was overall comparable in SSc patients and in previously described CHIP series (3)

In most SSc patients, we identified a single CHIP mutation. Several mutations were detected in two SSc patients: SETBP1 and NF1 in one and, TET2 and ATM in the other Clonal mutations included missense $(n=10)$, nonsense $(n=3)$, frameshift $(n=1)$ and a single splice site mutation. In all HD we detected a single CHIP mutation which occurred in DNMT3A, TP53 and CSF3R

Variant allele frequencies (VAF) of CHIP mutations ranged from 2 to $18.6 \%$ and did not differ between genes (DNMT3A or others). Mean age was the same in patients with DNMT3A mutations or with other mutations. However, $\mathrm{C}>\mathrm{T}$ transversions, that have been associated with ageing were more frequent in DNMT3A variants than in other genes, suggesting distinct mechanisms for mutation acquisition or clonal selection No major differences in clinical and laboratory data were observed between SSc patients with or without CHIP. SSc subtypes, disease duration, different organ involvements and the prevalence of ischemic events were not associated with the presence of CHIP, except less frequent pyrosis in patients with CHIP than those without. SSc patients with CHIP had significantly more anti-RNA polymerase III antibodies than those without CHIP $(p=0.045)$ At the time of analysis, 45 SSc patients had received a treatment for SSc which consisted in low-dose steroids, hydroxychloroquine, mycophenolate mofetil, cyclophosphamide or methotrexate. SSc patients with CHIP were significantly more exposed to cyclophosphamide ( $3 / 13$ vs. $3 / 77)(p=0.04)$ $(5,6.5$ and 11 gram respectively between 5 years to 8 years before the NGS sequencing analysis), but among these cyclophosphamide-exposed SSc the age was over 65 in $2 / 3$ of them. When considering all immunosuppressive drugs (cyclophosphamide, methotrexate and mycophenolate mofetil) SSc patients with CHIP were not more exposed than those without
CHIP $(p=0.75)$ No patient developed any hematologic malignancy and no cytopenia during the median follow-up of 13 months (0-24 months). One SSc patients with CHIP developed a small lung cancer few months after NGS testing.

Conclusion: Whether CHIP increases the risk to develop SSc or is a consequence of a SSc-derived modified bone marrow micro-environment remains to be explored.

Acknowledgments: na

Disclosure of Interests: None declared

DOI: 10.1136/annrheumdis-2020-eular.5489

$\mathrm{AB} 0162$

THE SIGNIFICANCE OF M1 AND M2 MONOCYTES IN SYSTEMIC SCLEROSIS

T. Michitsuij ${ }^{1}$, N. Iwamoto ${ }^{1}$, S. Tsuji ${ }^{1}$, A. Kawakami ${ }^{1} .{ }^{1}$ Department of Immunology and Rheumatology, Division of Advanced Preventive Medical Sciences, Nagasaki University Graduate School of Biomedical Sciences, Nagasaki, Japan, Nagasaki, Japan

Background: Recently, the relation between M2 macrophage and fibrosis have been reported in several diseases including systemic sclerosis (SSc). Similar with macrophages, monocytes can be classified into M1 and M2 subset, and the relation of imbalance of these monocytes with disease such as rheumatoid arthritis have been reported ${ }^{1,2}$.

Objectives: In this study, we attempted to investigate relationship among M1 or M2 monocytes in SSc.

Methods: This study included 23 SSc patients and 20 healthy donors. Using fluorescence-activated cell sorting, we defined CD14, CD68 and CCR2 positive cells as M1 monocytes and CD14, CX3CR1 and CD163 positive cells as M2 monocytes. We examined the ability of cytokines/chemokines secretion of CD14 positive cells from SSc by multiplex bead array assay using MAP human cytokine/chemokine Magnetic Bead Panel which can measure 38 cytokines/ chemokines. We next extracted M2 monocytes from CD14-positive cells using FACS, and we used the rest of the CD14 positive cells as M1-dominant monocytes. Then, we evaluated their ability of TGF- $\beta$ production by multiplex bead array assay.

Results: SSc patients had higher M2/M1 ratio as compared with healthy control ( 7.00 vs $1.63, \mathrm{P}<0.05)$. And, there was tendency that $\mathrm{M} 2 / \mathrm{M} 1$ ratio was highe in SSc patients complicated with interstitial pneumonia. Beads array analysis revealed that CCL4 and MCP-1 production from CD14 positive cells which consists $M 2>M 1$ (M2/M1 ratio>1) were higher than that from CD14 positive cells which consists $\mathrm{M} 2<\mathrm{M} 1$. Furthermore, the ability of TGF- $\beta$ secretion of M2 monocytes was higher than that of M1-dominant monocytes.

Conclusion: Our present study suggested that the imbalance of M1/M2 monocytes might contribute to pathogenesis of SSc.

References:

[1] Fadini GP, Simoni F, Cappellari R, et al. Pro-inflammatory monocyte-macrophage polarization imbalance in human hypercholesterolemia and atherosclerosis. Atherosclerosis 2014; 237(2): 805-8.

[2] Fukui S, Iwamoto N, Takatani A, et al. M1 and M2 Monocytes in Rheumatoid Arthritis: A Contribution of Imbalance of M1/M2 Monocytes to Osteoclastogenesis. Front Immunol 2017; 8: 1958.

Disclosure of Interests: None declared

DOI: 10.1136/annrheumdis-2020-eular.2773

\section{AB0163 ANTI-KU ANTIBODIES: MUCH MORE THAN SCLEROMYOSITIS}

L. Montolio-Chiva ${ }^{1}$, J. Narváez², F. Morandeira², J. Bas² ${ }^{2}$ C. Marco²,

X. González² , J. J. Alegre-Sancho ${ }^{1}$, E. Flores ${ }^{1}$, I. Vázquez-Gómez ${ }^{1}$, J.

M. López ${ }^{1}$, J. M. Nolla ${ }^{2} .{ }^{1}$ Universitary Peset Doctor Hospital, Valencia, Spain;

${ }^{2}$ Universitary Bellvitge Hospital, Barcelona, Spain

Background: Initially, anti-Ku antibodies (Ab) were described in patients with overlap syndrome with systemic sclerosis (SSc) and inflammatory myophaty (scleromyositis), although later they have been linked to a wide variety of systemic autoimmune diseases (SAD) questioning its diagnostic value. Recently, the possible existence of 2 different clinical phenotypes associated with these $A b$ has been described: one with myositis and high risk of intersticial lung disease (ILD) and another with positive anti-dsDNA Ab and glomerulonephritis.

Objectives: To analyze the clinical relevance and the main diagnosis of a serie of patients with positive anti-Ku Ab.

Methods: Descriptive observational study of patients with anti-Ku Ab in two third level hospitals between 2011 and 2019. Their determination was made at the criteria of the requesting physician.

Results: Twenty-three patients ( 20 women) with a median age of $59 \pm 14$ years (range, 24-83) and a follow up time (median) of 37 months (1-208) were identified. The main clinical and analytical characteristics, as well as the final clinica 
diagnosis of these patients are shown in Table 1. In the cluster analysis we could not identify clinical phenotypes, perhaps because of the small sample size. Only $50 \%$ of patients with myositis developed ILD. Regarding the final diagnosis, only 1 patient $(5 \%)$ was diagnosed of scleromyositis. Besides detecting them in patients with SSc (39\%) and idiopathic inflammatory myopathy (9\%), anti-Ku Ab were detected in other SAD, the most frequent were systemic lupus erythematosus, rheumatoid arthritis (RA) and overlap syndrome of SSc + RA.

Table 1. Main clinical-analytical manifestations and final diagnosis of pacientes with anti-Ku Ab.

\section{FINAL CLINICAL DIAGNOSIS}

Scleromyositis:

Idiopahtic inflammatory myopathy: 1

Systemic sclerosis (SSc): 6 (Pre-scleroderma: 3, limited SSc: 3)

Systemic lupus erythematosus: 2

Rheumatoid arthritis: 2

Overlap syndrome RA + limited SSc: 2

Primary Sjögren's syndrome: 1 (Secondary Sjögren's syndrome: 3 )

Mixed connective tissue disease: 1

Polymyalgia rheumatica: 1

Undifferentiated connective tissue disease: 1

Acute hepatitis due to HEV: 1

Autoimmune thrombocytopenia (ITP):

Drug-induced fibrosing ILD: 1

Systemic graft versus host disease (GVHD) in a patient with acute lymphoblastic leukemia

who received and autologous haematopoietic progenitor transplant: 1

Primary biliary cirrhosis: 1

CLINICAL MANIFESTATIONS (patients could have more than one):

Raynaud's phenomenon: $61 \%(14 / 23)$.

Inflammatory arthralgia/arthritis: $52 \%$ (12).

Lung involvement: $30.5 \%$ (7: NSIP 3, UIP 2, other patterns 2).

Serositis: $26 \%$ (6: pericarditis 4 , pleuritis 1 , pleuropericarditis 1 ).

Cardiac involvement: $26 \%$ (6: PHT by echocardiogram 3, myocarditis 2, arrhytmia 1).

Dry syndrome: $17 \%$ (4)

Myositis: $17 \%$ (4).

Esophageal involvement: $17 \%(4)$

Autoimmune cytopenias: leucolinfopenia: 17\% (4) / thrombocytopenia: 13\% (3).

Telangiectasias: $13 \%$ (3)

Photosensitivity: $13 \%$ (3).

Other: non-androgenic alopecia: $9 \%$ (2); sensory-motor polyneuropathy: $4.5 \%$ (1); Puffy

hands: $4.5 \%$ (1); fever: $4.5 \%$ (1); lymphadenitis: $4.5 \%$ (1); cold sores: $4.5 \%$ (1), and retinal hemorrhage: $4.5 \%$ (1).

\section{OTHER ASSOCIATED ANTIBODIES:}

ANA: $91 \%(21 / 23)$

anti Ro60/SSA Ab: $30.5 \%(7)$

Anti Ro52 Ab: 30.5\% (7)

Anti RNP Ab: $22 \%$ (5)

Anti-dsDNA: $17 \%(4)$

Anti-La/SSB Ab: $17 \%(4)$

Anticentromer $\mathrm{Ab}: 17 \%(4)$

Anti Mi-2 Ab: $13 \%$ (3)

Other: anti Sm Ab: $9 \%$ (2); anticardiolipin $\mathrm{Ab}(\mathrm{lgG}): 4.5 \%$ (1); PM/Scl: $4.5 \%$ (1); nucleosomes:

4.5\% (1); Scl70: 4.5\% (1); PL12: 4.5\% (1); anti-U1-RNP: $4.5 \%$ (1) and NOR90: $4.5 \%$ (1).

Conclusion: Anti-Ku Ab are related to a great variety of $\mathrm{SAD}$, without being a specific marker of any of them, nor being associated with any specific clinical manifestation. We couldn't confirm the existence of clinical phenotypes associated with the presence of these antibodies.

Disclosure of Interests: L Montolio-Chiva: None declared, J. Narváez: None declared, Francisco Morandeira: None declared, Jordi Bas: None declared, Carla Marco: None declared, Xavier González: None declared, Juanjo J Alegre-Sancho Consultant of: UCB, Roche, Sanofi, Boehringer, Celltrion, Paid instructor for: GSK, Speakers bureau: MSD, GSK, Lilly, Sanofi, Roche, UCB, Actelion, Pfizer, Abbvie, Novartis, Eduardo Flores: None declared, I Vázquez-Gómez: None declared, Jose María López: None declared, Joan Miquel Nolla: None declared DOI: 10.1136/annrheumdis-2020-eular.3858

\section{AB0164 ACTIVITIES OF THE OXIDATIVE-RELATED ENZYMES IN SYSTEMIC SCLEROSIS}

E. Mozgovaya ${ }^{1}$, I. Zborovskaya ${ }^{1}$, S. Bedina ${ }^{1}$, A. Trofimenko ${ }^{1}$, M. Mamus ${ }^{1}$, E. Tikhomirova ${ }^{1}$, S. Spitsina ${ }^{1} .{ }^{1}$ Research Institute of Clinical and Experimental Rheumatology named after A.B. Zborovsky, Volgograd, Russian Federation

Background: The oxidative-related enzymes are involved in the pathogenesis of various stages of systemic sclerosis (SSc). SSc is a chronic autoimmune disorder that is intimately associated with vascular damage and therefore with chronic perfusion/reperfusion and oxidative organ injury. Mesenchymal cell activation in SSc is now also considered to be mediated primarily through oxidative burst. Regulation of oxidative stress by specific enzymes including several purine metabolism enzymes is likely to play an important role in SSc progression.

Objectives: to characterize interrelationships among circulating xanthine oxidase $(\mathrm{XO})$, xanthine dehydrogenase $(\mathrm{XDH})$, superoxide dismutase (SOD) activities and SSc activity.
Methods: The study was performed according to bioethical standards. 51 patients with verified SSc and 30 healthy controls were included in the study. The diagnosis was verified according to ACR/EULAR 2013 criteria. We assessed SSc activity in compliance with the original activity scale that is commonly used in Russia [Guseva N.G., 1993] and by the 2001 European Scleroderma Study Group Activity Index. XO (EC 1.17.3.2), XDH (EC 1.17.1.4), and SOD (EC 1.15.1.1) plasma activities were measured using spectrophotometric techniques as previously described [Dubinina E.E., 1986; Karpova O.V., 2006]. Results are expressed as mean $\pm S D$. The Mann-Whitney $U$ test and Spearman's correlation coefficient were used for statistical analysis.

Results: Mean age of patients was $42.8 \pm 1.3$ years, mean SSc duration was $7.9 \pm 0.7$ years. Mean enzymatic activities in normal controls were $3.43 \pm 0.56 \mathrm{nmol} /$ $\mathrm{ml} \mathrm{min} \mathrm{(for} \mathrm{XO),} 5.19 \pm 0.71 \mathrm{nmol} / \mathrm{ml} \mathrm{min}$ (for XDH), and $5.40 \pm 1.03$ units (for SOD). The respective enzymatic activities in SSc group were $3.91 \pm 0.62 \mathrm{nmol} / \mathrm{ml} \mathrm{min}$ $7.10 \pm 0.71 \mathrm{nmol} / \mathrm{ml} \mathrm{min}$, and $7.10 \pm 2.19$ units. All these mean activities were significantly higher in SSc patients comparing to healthy individuals $(p<0.001)$. XO and $\mathrm{XDH}$ activities positively correlated with $\mathrm{SSc}$ activity $(r=0.499, \mathrm{p}<0.001$, and $r=0.741, p<0.001$, respectively). The opposite but weaker trend was observed for SOD activity and SSc disease activity $(r=-0.190, p=0.188)$.

Conclusion: $\mathrm{SSc}$ is characterized by an increase in the intensity of oxidative and antioxidant processes, more pronounced in high disease activity. A close relationship between function of prooxidant/antioxidant enzymes and some of the key SSc pathogenetic mechanisms, especially vascular disease and fibroblast activation, is widely considered. Overall increase of oxidative stress in patients with higher disease activity, as well as depletion of antioxidant capacity can be also linked with disturbance of purine metabolism through XO and XDH modulation. Pathogenetic influence of this imbalance can also be mediated through initial phase of neutrophil extracellular traps (NETs) formation, an eventual source of nucleoprotein containing autoepitopes.

Disclosure of Interests: None declared

DOI: 10.1136/annrheumdis-2020-eular.3462

\section{AB0165 THE ROLE OF CXCL4, CXCL8 AND GDF-15 IN SYSTEMIC SCLEROSIS}

J. E. Oller Rodriguez ${ }^{1}$, E. Grau García ${ }^{1}$, R. Gonzalez Mazario ${ }^{1}$, M. De la Rubia Navarro $^{1}$, C. Pávez Perales ${ }^{1}$, S. Leal Rodriguez ${ }^{1}$, I. Martínez Cordellat ${ }^{1}$, F. M. Ortiz Sanjuan ${ }^{1}$, E. Vicens Bernabeu ${ }^{1}$, R. Negueroles Albuixech ${ }^{1}$, I. Chalmeta Verdejo $^{1}$, C. Alcañiz Escandell ${ }^{1}$, J. J. Fragio-Gill ${ }^{1}$, I. Cánovas Olmos ${ }^{1}$, C. Nájera Herranz $^{1}$, L. Gonzalez Puig ${ }^{1}$, J. Ivorra Cortés ${ }^{1}$, D. Hervás Marín ${ }^{2}$, J. A. Román Ivorra ${ }^{1} .{ }^{1}$ Rheumatology Service. HUP La Fe., Valencia., Spain; ${ }^{2}$ Biostatistics Unit. IIS La Fe, Valencia, Spain

Background: Systemic Sclerosis (SSc) is an autoinmune disease that can affect several organs and its mortality is fundamentally related to its pulmonary involvement.

It is mandatory to seek for biomarkers that help us with early diagnosis and that are also useful for predicting organic involvement, so that we can adjust the diagnostic and therapeutic approach.

Objectives: Our aim was to check if the presence of CXCL4, CXCL8 and GDF15 is greater in the disease than in healthy population, and also their involvement in organic damage.

Methods: Observational and cross-sectional study, with a prospectively performed protocol, of patients diagnosed of SSc according to ACR/EULAR 2013 criteria. Demographic, clinical, analytical, activity (EUSTAR index), severity (Medsger scale and modified Rodnan index), health perception (SF36) and disability (HAQ and Cochin test) variables were collected. Moreover, Videocapillaroscopy (VCL) and Respiratory Function Test were made, as well High ResoIution Lung Tomography and Echocardiography in order to describe pulmonary features. Serum levels of CXCL4, CXCL8 and GDF-15 were measured in SSc patients and in healthy controls.

Results: A total of 42 patients (95.4\% women) were included, with an average age of 59.2 years. The median of years since diagnosis was 4 , by 6 since the first non-Raynaud symptom. 20 patients were diagnosed with limited SSc, 20 patients diffusely and 2 patients with SSc without scleroderma. 42 healthy controls were also included.

We found significantly higher levels of GDF-15 in patients with $\mathrm{SSc}(\mathrm{P}<0.001)$, without significant differences in $\mathrm{CXCL} 4$ and $\mathrm{CXCL} 8$ levels between patients with SSc and healthy controls.

The presence of GDF-15 was associated with diffuse SSc $(P=0.009)$, pulmonary arterial hypertension $(P=0.038)$, interstitial lung disease $(P=0.004)$, decreased forced vital capacity (FVC), ( $P=0.002)$, high serum titles of antiScl70 ( $P=0.006)$ increased disease activity measured by EUSTAR index $(P=0.001)$, as with capillary dilations in Capillaroscopy $(\mathrm{P}=0.015)$

Moreover, we found an association between CXCL4 levels and the consumption of complement $\mathrm{C} 3$ fraction $(\mathrm{P}=0.008)$ and skin involvement (higher Rodnan modified score), $(P=0.001)$; not finding association with lung involvement or other features (spirometric or analytical changes, capillaroscopy or functional tests) 